IJMMS 25:10 (2001) 629-635

PII. S0161171201005191

http://ijmms.hindawi.com

(C) Hindawi Publishing Corp.

\title{
ON THE RATE OF CONVERGENCE OF BOOTSTRAPPED MEANS IN A BANACH SPACE
}

\author{
S. EJAZ AHMED, T.-C. HU, and ANDREI I. VOLODIN \\ (Received 5 January 2000 and in revised form 5 May 2000)
}

\begin{abstract}
We establish the complete convergence for arrays of Banach space valued random elements. This result is applied to bootstrapped means of random elements to obtain their strong consistency and is derived in the spirit of Baum-Katz/Hsu-Robbins/Spitzer type convergence.
\end{abstract}

2000 Mathematics Subject Classification. Primary 60B12; Secondary 60F15.

1. Introduction. The main focus of the present investigation is the determination of the rates of convergence for strong laws of large numbers for arrays of random elements. Interestingly, this result will be applied to establish the strong consistency for bootstrapped means taking values in Banach spaces. More precisely, we present Chung type strong law of large numbers for arrays of rowwise independent random elements under conditions similar to those given by Bozorgnia et al. [1]; Hu et al. [3]; and Sung [6]. This result is of interest since it holds for an arbitrary real separable Banach space without imposing any geometric conditions. Thus, the results of this paper are more general than those presented in the papers cited above.

Strong laws of large numbers are of practical use in establishing the strong asymptotic validity of the bootstrapped mean for random elements. Furthermore, they are of considerable theoretical and practical interest in investigating the consistency of bootstrap estimators.

Some results on the consistency of the bootstrapped mean of random elements in Banach spaces are given in [1]. However, these consistency results impose a geometric condition (Rademacher type $p$ ) on the Banach space. Moreover, the random elements from the original sample are assumed to be independently and identically distributed (i.i.d.). However, in the present investigation we do not make any assumptions regarding the marginal or joint distributions of the random elements forming the sample and the random elements assuming values in an arbitrary real separable Banach space. A similar situation was considered for real-valued random variables by Li et al. [4, Theorem 2.1].

In order to obtain strong consistency for the bootstrapped mean, we assume the corresponding weak consistency. In this case, the main result of Bozorgnia et al. [1] can be seen as a special case of the results given in Theorem 3.2 of this paper.

2. Chung's type strong law of large numbers. First, we state the following recent theorem which forms the basis of our results. 
THEOREM 2.1 (see [2, Theorem 3.2]). Let $\left\{k_{n}, n \geq 1\right\}$ be a sequence of positive integers, let $\left\{Y_{n k}, 1 \leq k \leq k_{n}, n \geq 1\right\}$ be an array of rowwise independent random elements taking values in a real separable Banach space, and let $\left\{c_{n}, n \geq 1\right\}$ be a sequence of positive constants. Suppose that $E\left\|Y_{n k}\right\|^{q}<\infty, 1 \leq k \leq k_{n}, n \geq 1$ for some $0<q \leq 2$.

Moreover, assume that

(1) $\sum_{n=1}^{\infty} c_{n} \sum_{k=1}^{k_{n}} P\left\{\left\|Y_{n k}\right\|>\epsilon\right\}<\infty$ for all $\epsilon>0$,

(2) $\sum_{k=1}^{k_{n}} Y_{n k} \stackrel{P}{\rightarrow} 0$,

(3) there exists $J \geq 1$ such that

$$
\sum_{n=1}^{\infty} c_{n}\left(\sum_{k=1}^{k_{n}} E\left\|Y_{n k}\right\|^{q}\right)^{J}<\infty
$$

(4) $\sum_{k=1}^{k_{n}} P\left\{\left\|Y_{n k}\right\|>\delta\right\}=o$ (1) for some $\delta>0$ as $n \rightarrow \infty$ if $\liminf _{n \rightarrow \infty} c_{n}=0$. Then

$$
\sum_{n=1}^{\infty} c_{n} P\left\{\left\|\sum_{k=1}^{k_{n}} Y_{n k}\right\|>\epsilon\right\}<\infty \quad \forall \epsilon>0 .
$$

Recently, Bozorgnia et al. [1], Hu et al. [3], and Sung [6] proved Chung's type strong laws of large numbers for arrays of rowwise independent random variables or random elements. We now apply Theorem 2.1 to obtain a similar result in a general real separable Banach space under the assumption that the corresponding weak law of large numbers holds. Theorem 2.2 is an adaptation of Theorem 2.1.

THEOREM 2.2. Let $\left\{Z_{n k}, 1 \leq k \leq k_{n}, n \geq 1\right\}$ be an array of rowwise independent random elements taking values in a real separable Banach space and let $\left\{a_{n}, n \geq 1\right\}$ and $\left\{c_{n}, n \geq 1\right\}$ be sequences of positive constants. Suppose that $E \psi\left(\left\|Z_{n k}\right\|\right)<\infty$ and $E\left\|Z_{n k}\right\|^{q}<\infty$ for some $0<q \leq 2$ and some continuous nondecreasing function $\psi$ : $\mathbb{R}^{+} \rightarrow \mathbb{R}^{+}$such that, for all $\epsilon>0$,

$$
\sup _{n \geq 1} \frac{\psi\left(a_{n}\right)}{\psi\left(\epsilon a_{n}\right)}<\infty
$$

Then the conditions

(i) $\sum_{n=1}^{\infty}\left(c_{n} / \psi\left(a_{n}\right)\right) \sum_{k=1}^{k_{n}} E \psi\left(\left\|Z_{n k}\right\|\right)<\infty$,

(ii) $\left(1 / a_{n}\right) \sum_{k=1}^{k_{n}} Z_{n k} \stackrel{P}{\rightarrow} 0$,

(iii) there exists $J \geq 1$ such that

$$
\sum_{n=1}^{\infty} \frac{c_{n}}{a_{n}^{q J}}\left(\sum_{k=1}^{k_{n}} E\left\|Z_{n k}\right\|^{q}\right)^{J}<\infty,
$$

(iv) $\left(c_{n} / \psi\left(a_{n}\right)\right) \sum_{k=1}^{k_{n}} E \psi\left(\left\|Z_{n k}\right\|\right)=o(1)$ as $n \rightarrow \infty$ if $\liminf _{n \rightarrow \infty} c_{n}=0$, imply that

$$
\sum_{n=1}^{\infty} c_{n} P\left\{\left\|\sum_{k=1}^{k_{n}} Z_{n k}\right\|>\epsilon a_{n}\right\}<\infty \quad \forall \epsilon>0 .
$$


Proof. The assumptions of Theorem 2.1 for the array $\left\{Y_{n k}=Z_{n k} / a_{n}, 1 \leq k \leq k_{n}\right.$, $n \geq 1\}$ can be rewritten as follows.

(1) $\sum_{n=1}^{\infty} c_{n} \sum_{k=1}^{k_{n}} P\left\{\left\|Z_{n k}\right\|>\epsilon a_{n}\right\}<\infty$ for all $\epsilon>0$,

(2) $\left(1 / a_{n}\right) \sum_{k=1}^{k_{n}} Z_{n k} \stackrel{P}{\rightarrow} 0$,

(3) There exists $J \geq 1$ such that

$$
\sum_{n=1}^{\infty} \frac{c_{n}}{a_{n}^{q J}}\left(\sum_{k=1}^{k_{n}} E\left\|Z_{n k}\right\|^{q}\right)^{J}<\infty
$$

(4) $\sum_{k=1}^{k_{n}} P\left\{\left\|Y_{n k}\right\|>\delta a_{n}\right\}=o(1)$ as $n \rightarrow \infty$ if $\liminf _{n \rightarrow \infty} c_{n}=0$.

Note that conditions (ii) and (2) are the same and that (iii) is equivalent to (3). For (i) and (iv), using Markov's inequality and the assumptions on the function $\psi$, we have the following:

$$
P\left\{\left\|Z_{n k}\right\|>\epsilon a_{n}\right\} \leq \frac{1}{\psi\left(\epsilon a_{n}\right)} E \psi\left(\left\|Z_{n k}\right\|\right) \leq \frac{C(\epsilon)}{\psi\left(a_{n}\right)} E \psi\left(\left\|Z_{n k}\right\|\right) .
$$

It should be pointed out that the assumptions on the function $\psi(\cdot)$ seem to be the most natural and general for the current work.

3. The consistency of the bootstrapped mean. We now outline the bootstrap procedure. Let $\left\{X_{n} ; n \geq 1\right\}$ be a sequence of (not necessarily independent or identically distributed) random elements defined on some complete probability space $(\Omega, \mathscr{F}, P)$ which take values in a real separable Banach space. For $\omega \in \Omega$ and $n \geq 1$, let $P_{n}(\omega)=$ $n^{-1} \sum_{i=1}^{n} \delta_{X_{i}(\omega)}$ denote the empirical measure. For $n \geq 1$, let $\left\{\hat{X}_{n, j}^{\omega} ; 1 \leq j \leq k_{n}\right\}$ be i.i.d. random elements with law $P_{n}(\omega)$, where $k_{n}$ is a positive integer. Let $\bar{X}_{n}(\omega)$ denote the sample mean of $\left\{X_{i}(\omega) ; 1 \leq i \leq n\right\}, n \geq 1$, that is, $\bar{X}_{n}(\omega)=(1 / n) \sum_{i=1}^{n} X_{i}(\omega)$.

To prove the consistency of bootstrapped mean, we use the following lemma. We formulated this simple observation as a lemma since it is frequently applied in the proof.

LEMMA 3.1. If $s>0$, then for almost every $\omega \in \Omega$,

$$
E\left\|\hat{X}_{n, 1}^{\omega}-\bar{X}_{n}(\omega)\right\|^{s} \leq A_{s}\left[\frac{1}{n} \sum_{i=1}^{n}\left\|X_{i}(\omega)\right\|^{s}+\left\|\bar{X}_{n}(\omega)\right\|^{s}\right],
$$

where $A_{s}=2^{s-1}$ for $s \geq 1$ and $A_{s}=1$ for $0<s<1$.

Proof. For almost every $\omega \in \Omega$,

$$
E\left\|\hat{X}_{n, j}^{\omega}-\bar{X}_{n}(\omega)\right\|^{s}=\frac{1}{n} \sum_{i=1}^{n}\left\|X_{i}(\omega)-\bar{X}_{n}(\omega)\right\|^{s} \leq A_{s}\left[\frac{1}{n} \sum_{i=1}^{n}\left(\left\|X_{i}(\omega)\right\|^{s}+\left\|\bar{X}_{n}(\omega)\right\|^{s}\right)\right],
$$

by the $c_{r}$-inequalities (Loève [5, page 157]).

We can now prove the main application presented in this paper.

THEOREM 3.2. Let $\left\{X_{n}, n \geq 1\right\}$ be a sequence of random elements taking values in a real separable Banach space and let $\left\{a_{n}, n \geq 1\right\},\left\{b_{n}, n \geq 1\right\},\left\{c_{n}, n \geq 1\right\}$, and $\left\{d_{n}, n \geq 1\right\}$ be sequences of positive constants. Suppose that there exists $0<q \leq 2$ and $r \geq q$ 
such that

(1) $\sup _{n \geq 1}\left(1 / d_{n}\right)\left\|\bar{X}_{n}\right\|<\infty$ a.s. and $\sup _{n \geq 1}\left(1 / b_{n}\right) \sum_{i=1}^{n}\left\|X_{i}\right\|^{q}<\infty$ a.s.,

(2) $\sum_{n=1}^{\infty}\left(c_{n} / a_{n}^{r}\right) k_{n}^{r / q} d_{n}^{r}<\infty$ and $\sum_{n=1}^{\infty}\left(c_{n} / a_{n}^{r}\right) b_{n}^{r / q} \max \left\{k_{n} / n,\left(k_{n} / n\right)^{r / q}\right\}<\infty$,

(3) The bootstrapped mean is weakly consistent, that is, for almost every $\omega \in \Omega$,

$$
\frac{1}{a_{n}}\left\|\sum_{k=1}^{k_{n}}\left(\hat{X}_{n, k}^{\omega}-\bar{X}_{n}(\omega)\right)\right\| \stackrel{P}{\longrightarrow} 0,
$$

(4) $c_{n}\left(b_{n}^{r / a} k_{n} / a_{n}^{r} n\right) \rightarrow 0$ and $c_{n}\left(k_{n} d_{n}^{r}\right) / a_{n}^{r} \rightarrow 0$ if $\liminf _{n \rightarrow \infty} c_{n}=0$.

Then the bootstrapped mean is strongly consistent, that is, for almost every $\omega \in \Omega$ and for all $\epsilon>0$,

$$
\sum_{n=1}^{\infty} c_{n} P\left\{\left\|\sum_{k=1}^{k_{n}}\left(\hat{X}_{n, k}^{\omega}-\bar{X}_{n}(\omega)\right)\right\|>\epsilon a_{n}\right\}<\infty .
$$

Proof. We need only check conditions (i)-(v) of Theorem 2.2 for the array $\left\{Z_{n k}=\hat{X}_{n, k}^{\omega}-\bar{X}_{n}(\omega), 1 \leq k \leq k_{n}, n \geq 1\right\}$ with $\psi(t)=t^{r}, t \geq 0$.

For (i), an application of Lemma 3.1 with $s=r$ yields, for almost every $\omega \in \Omega$,

$$
\begin{aligned}
& \sum_{n=1}^{\infty} \frac{c_{n}}{\psi\left(a_{n}\right)} \sum_{k=1}^{k_{n}} E \psi\left(\left\|Z_{n k}\right\|\right) \\
& \quad=\sum_{n=1}^{\infty} \frac{c_{n}}{a_{n}^{r}} \sum_{k=1}^{k_{n}} E\left\|\hat{X}_{n, k}^{\omega}-\bar{X}_{n}(\omega)\right\|^{r} \\
& \quad \leq A_{r}\left(\sum_{n=1}^{\infty} \frac{c_{n} k_{n}}{a_{n}^{r} n} \sum_{i=1}^{n}\left\|X_{i}(\omega)\right\|^{r}+\sum_{n=1}^{\infty} \frac{c_{n} k_{n}}{a_{n}^{r}}\left\|\bar{X}_{n}(\omega)\right\|^{r}\right) \\
& \quad \leq A_{r}\left(\sum_{n=1}^{\infty} \frac{c_{n} k_{n} b_{n}^{r / q}}{a_{n}^{r} n}\left[\frac{1}{b_{n}} \sum_{i=1}^{n}\left\|X_{i}(\omega)\right\|^{q}\right]^{r / q}+\sum_{n=1}^{\infty} \frac{c_{n} k_{n} d_{n}^{r}}{a_{n}^{r}}\left[\frac{1}{d_{n}}\left\|\bar{X}_{n}(\omega)\right\|\right]^{r}\right)<\infty
\end{aligned}
$$

by (1) and (2) and since $k_{n} \leq k_{n}^{r / q}$.

For (ii), let $J=r / q \geq 1$. Another application of Lemma 3.1 with $s=q$ yields, for almost every $\omega \in \Omega$,

$$
\begin{aligned}
& \sum_{n=1}^{\infty} \frac{c_{n}}{a_{n}^{q J}}\left(\sum_{k=1}^{k_{n}} E\left\|\hat{X}_{n, k}^{\omega}-\bar{X}_{n}(\omega)\right\|^{q}\right)^{J} \\
& \leq A_{q}^{J} 2^{J-1}\left(\sum_{n=1}^{\infty} \frac{c_{n}}{a_{n}^{r}}\left(\frac{k_{n}}{n}\right)^{r / q}\left[\sum_{i=1}^{n}\left\|X_{i}(\omega)\right\|^{q}\right]^{r / q}+\sum_{n=1}^{\infty} \frac{c_{n} k_{n}^{r / q}}{a_{n}^{r}}\left\|\bar{X}_{n}(\omega)\right\|^{r}\right) \\
& \quad \leq A_{q}^{J}\left(\sum_{n=1}^{\infty} \frac{c_{n} b_{n}^{r / q}}{a_{n}^{r}}\left(\frac{k_{n}}{n}\right)^{r / q}\left[\frac{1}{b_{n}} \sum_{i=1}^{n}\left\|X_{i}(\omega)\right\|^{q}\right]^{r / q}+\sum_{n=1}^{\infty} c_{n} k_{n}^{r / q}\left(\frac{d_{n}}{a_{n}}\right)^{r}\left(\frac{1}{d_{n}}\left\|\bar{X}_{n}(\omega)\right\|\right)^{r}\right)<\infty,
\end{aligned}
$$

by (1) and (2). 
Since (iii) is the same as (3), it is automatically satisfied. Now, considering (iv), by another application of the lemma with $s=r$ and by the same argument as in the proof of (i), we have, for almost every $\omega \in \Omega$,

$$
\begin{aligned}
\frac{c_{n}}{\psi\left(a_{n}\right)} & \sum_{k=1}^{k_{n}} E \psi\left(\left\|Z_{n k}\right\|\right) \\
& =\frac{c_{n}}{a_{n}^{r}} \sum_{k=1}^{k_{n}} E\left\|\hat{X}_{n, k}^{\omega}-\bar{X}_{n}(\omega)\right\|^{r} \\
& \leq A_{r}\left(\frac{c_{n} k_{n}}{a_{n}^{r} n} \sum_{i=1}^{n}\left\|X_{i}(\omega)\right\|^{r}+\frac{c_{n} k_{n}}{a_{n}^{r}}\left\|\bar{X}_{n}(\omega)\right\|^{r}\right) \\
& \leq A_{s}\left(c_{n} \frac{k_{n} b_{n}^{r / q}}{a_{n}^{r} n}\left(\frac{1}{b_{n}} \sum_{i=1}^{n}\left\|X_{i}(\omega)\right\|^{q}\right)^{r / q}+c_{n} \frac{k_{n} d_{n}^{r}}{a_{n}^{r}}\left(\frac{1}{d_{n}}\left\|\bar{X}_{n}(\omega)\right\|\right)^{r}\right) \rightarrow 0
\end{aligned}
$$

in case $\liminf _{n \rightarrow \infty} c_{n}=0$, by (4) and (1).

It is natural to question whether the conditions of Theorem 3.2 can be easily verified. We give an example verifying the conditions of Theorem 3.2 by providing an alternative proof of the main result of Bozorgnia et al. [1, Theorem 3.1]. This proof is substantially simpler than the original one.

We recall that a Banach space is said to be of type $q, 1 \leq q \leq 2$, if there exists a constant $C>0$ such that, for any sequence $X_{1}, \ldots, X_{n}$ of independent, mean zero random elements taking values in the Banach space, the following inequality holds:

$$
E\left\|\sum_{i=1}^{n} X_{i}\right\|^{q} \leq C \sum_{i=1}^{n} E\left\|X_{i}\right\|^{q} .
$$

COROLlary 3.3. Let $\left\{X, X_{n}, n \geq 1\right\}$ be i.i.d. random elements taking values in a real separable Banach space of type $q, 1<q \leq 2$ with $E\|X\|^{q}<\infty$ and $E X=\mu$. Then for all $\epsilon>0$ and for almost every $\omega \in \Omega$,

$$
\sum_{n=1}^{\infty} P\left\{\left\|\sum_{k=1}^{n}\left(\hat{X}_{n, k}^{\omega}-\mu\right)\right\|>\epsilon n\right\}<\infty .
$$

Proof. The first step is to prove that, for almost every $\omega \in \Omega$,

$$
\sum_{n=1}^{\infty} P\left\{\left\|\sum_{k=1}^{n}\left(\hat{X}_{n, k}^{\omega}-\bar{X}_{n}(\omega)\right)\right\|>\epsilon n\right\}<\infty .
$$

In order to establish this fact, we need to prove that the assumptions of Theorem 3.2 are satisfied. To this end, let $a_{n}=b_{n}=k_{n}=n, c_{n}=d_{n}=1$ and $r>(q / q-1)$. It is easy then to see that (2) is satisfied. Furthermore, there is no need to check (4). However, for (1) we note that $\bar{X}_{n}(\omega) \rightarrow \mu$ a.s. by Mourier's strong law of large numbers in Banach spaces and $(1 / n) \sum_{i=1}^{n}\left\|X_{i}\right\|^{q} \rightarrow E\|X\|^{q}$ a.s. by the ordinary (or Kolmogorov) strong law of large numbers. 
In order to obtain weak consistency (3), we apply the Markov inequality and use the fact that the Banach space is of type $q$. So, for almost every $\omega \in \Omega$,

$$
\begin{aligned}
P\left\{\left\|\sum_{k=1}^{n}\left(\hat{X}_{n, k}^{\omega}-\bar{X}_{n}(\omega)\right)\right\|>\epsilon n\right\} & \leq \frac{1}{\epsilon^{q} n^{q}} E\left\|\sum_{k=1}^{n}\left(\hat{X}_{n, k}^{\omega}-\bar{X}_{n}(\omega)\right)\right\|^{q} \\
& \leq \frac{C}{\epsilon^{q} n^{q}} n E\left\|\hat{X}_{n, 1}^{\omega}-\bar{X}_{n}(\omega)\right\|^{q} \\
& \leq \frac{C A_{q}}{\epsilon^{q} n^{q-1}}\left[\frac{1}{n} \sum_{i=1}^{n}\left\|X_{i}(\omega)\right\|^{q}+\left\|\bar{X}_{n}(\omega)\right\|^{q}\right]
\end{aligned}
$$

again by an application of Lemma 3.1 with $s=q$. So, $P\left\{\left\|\sum_{k=1}^{n}\left(\hat{X}_{n, j}^{\omega}-\bar{X}_{n}(\omega)\right)\right\|>\epsilon n\right\} \rightarrow$ 0 almost every $\omega \in \Omega$, by the same argument as in the proof of (1).

The next step is to prove that $\sum_{n=1}^{\infty} P\left\{\left\|\sum_{k=1}^{n}\left(\hat{X}_{n, k}^{\omega}-\mu\right)\right\|>\epsilon n\right\}<\infty$ for almost every $\omega \in \Omega$. It suffices to note that since $\bar{X}_{n}(\omega) \rightarrow \mu$ a.s., for almost every $\omega \in \Omega$, there exists an integer $N=N(\omega)$ such that for all $n>N$ we have $\left\|\bar{X}_{n}(\omega)-\mu\right\|<\epsilon / 2$. Let $n>N$, then

$$
\begin{aligned}
P\left\{\left\|\sum_{k=1}^{n}\left(\hat{X}_{n, k}^{\omega}-\mu\right)\right\|>\epsilon n\right\} & =P\left\{\left\|\left[\frac{1}{n} \sum_{k=1}^{n}\left(\hat{X}_{n, k}^{\omega}-\bar{X}_{n}(\omega)\right)\right]+\left[\bar{X}_{n}(\omega)-\mu\right]\right\|>\epsilon\right\} \\
& \leq P\left\{\left\|\sum_{k=1}^{n}\left(\hat{X}_{n, k}^{\omega}-\bar{X}_{n}(\omega)\right)\right\|>\frac{\epsilon n}{2}\right\} .
\end{aligned}
$$

REMARK 3.4. (1) Theorems 2.1, 2.2, and 3.2 are trivial in case $\sum_{n=1}^{\infty} c_{n}<\infty$.

(2) We do not consider the case $q=1$ in the corollary since our proof will not cover this situation.

(3) Finally, we are not able to compare Theorem 3.2 of this paper and Theorem 2.1 of Li et al. [4] since their assumptions concern convergence of partial sums, whereas we use only boundedness of partial sums.

ACKNOWLEDGEMENT. The authors are grateful to the referee for carefully reading the manuscript and for providing some comments which helped improve the presentation. The work of S. E. Ahmed is supported by a grant from the Natural Sciences and Engineering Research Council of Canada.

\section{REFERENCES}

[1] A. Bozorgnia, R. F. Patterson, and R. L. Taylor, Chung type strong laws for arrays of random elements and bootstrapping, Stochastic Anal. Appl. 15 (1997), no. 5, 651-669. MR 98m:60042. Zbl 899.60028.

[2] T.-C. Hu, A. Rosalsky, D. Szynal, and A. I. Volodin, On complete convergence for arrays of rowwise independent random elements in Banach spaces, Stochastic Anal. Appl. 17 (1999), no. 6, 963-992. CMP 1721 930. Zbl 940.60032.

[3] T.-C. Hu and R. L. Taylor, On the strong law for arrays and for the bootstrap mean and variance, Int. J. Math. Math. Sci. 20 (1997), no. 2, 375-382. MR 97k:60011. Zbl 883.60024.

[4] D. Li, A. Rosalsky, and S. E. Ahmed, Complete convergence of bootstrapped means and moments of the supremum of normed bootstrapped sums, Stochastic Anal. Appl. 17 (1999), no. 5, 799-814. CMP 1714 899. Zbl 990.78202.

[5] M. Loève, Probability Theory. I, 4th ed., Graduate Texts in Mathematics, vol. 45, SpringerVerlag, Heidelberg, 1977. MR 58\#31324a. Zbl 359.60001. 
[6] S. H. Sung, Complete convergence for sums of arrays of random elements, Int. J. Math. Math. Sci. 23 (2000), no. 11, 789-794. CMP 1764121.

S. EJAZ Ahmed: Department of MAthematics and Statistics, University of Regina, REgina, SASKATCHEWAN, CANADA S4S 0A2

E-mail address: ahmed@math . uregina.ca

T.-C. Hu: Department of Mathematics, National TSING Hua University, HSinchu 300, TAIWAN

ANDREI I. VOLODIN: DEPARTMENT OF MATHEMATICS AND STATISTICS, UNIVERSITY OF REGINA, REgina, SASKatcheWAN, CANADA S4S 0A2

E-mail address: volodin@math. uregina.ca 


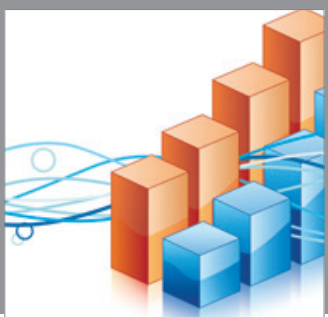

Advances in

Operations Research

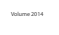

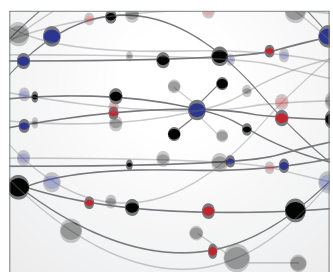

\section{The Scientific} World Journal
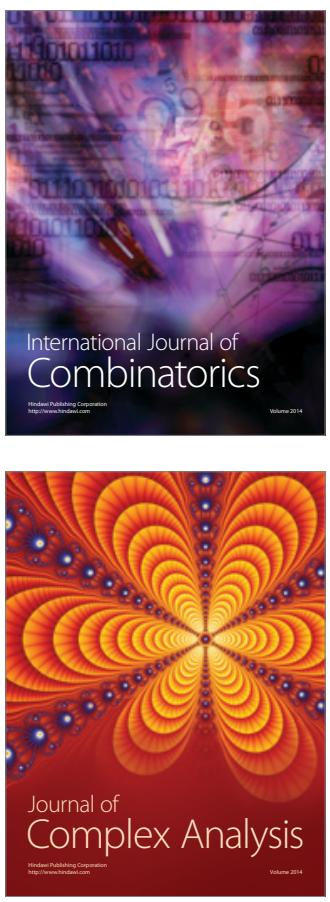

International Journal of

Mathematics and

Mathematical

Sciences
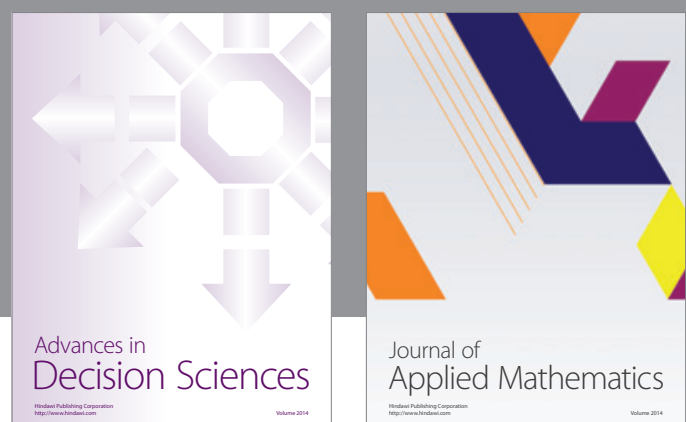

Journal of

Applied Mathematics
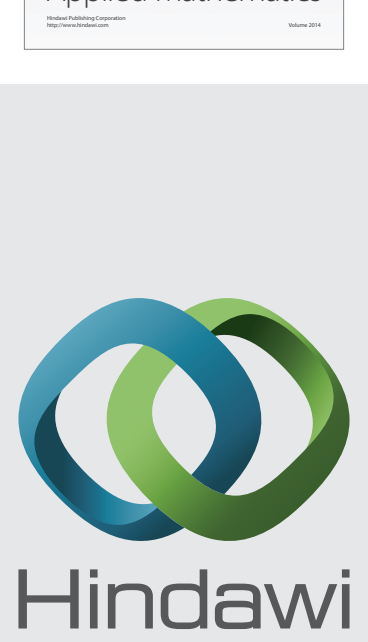

Submit your manuscripts at http://www.hindawi.com
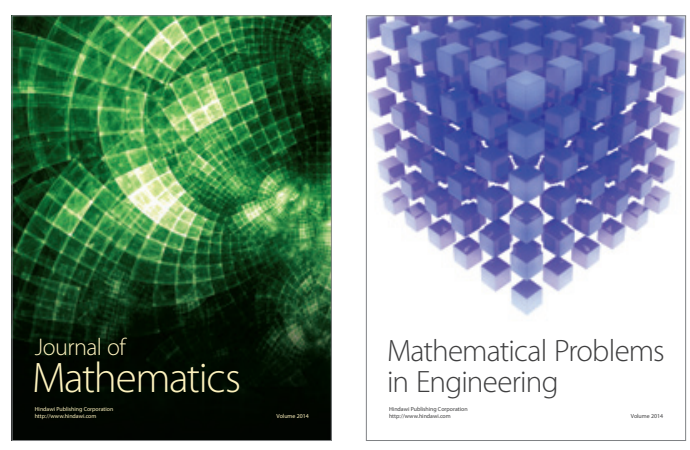

Mathematical Problems in Engineering
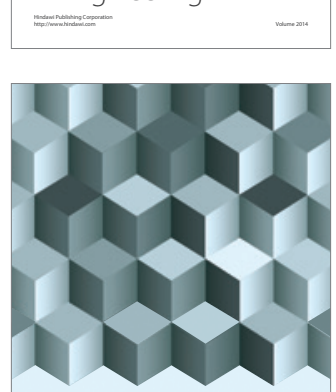

Journal of

Function Spaces
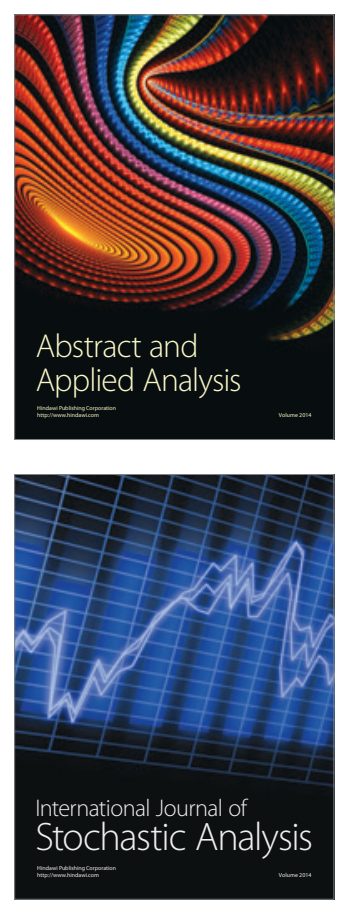

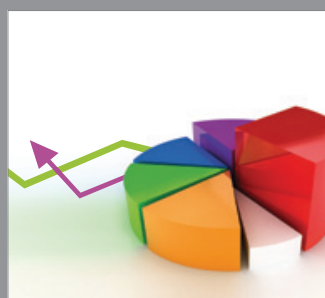

ournal of

Probability and Statistics

Promensencen
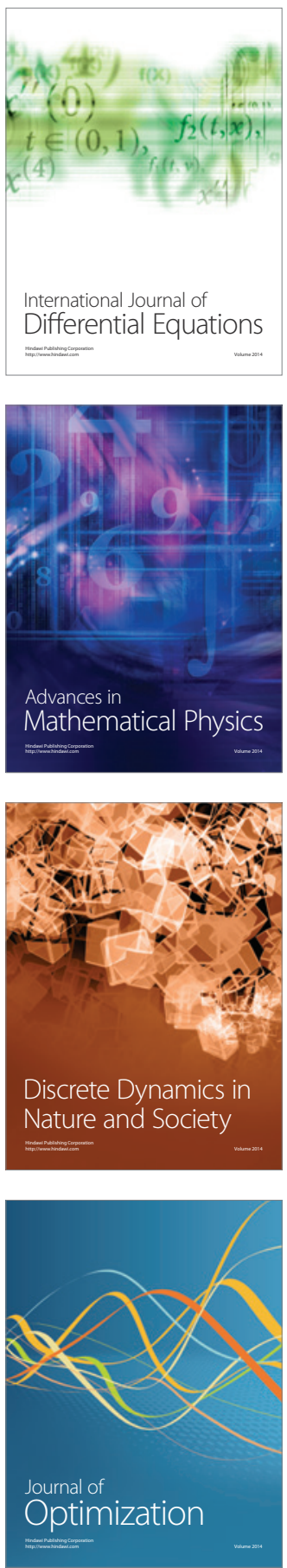